\title{
ACE-Hemmer im 1. Schwangerschafts-Trimenon unbedenklich
}

\section{Das erhöhte Fehlbildungsrisiko bei Einnahme von ACE-Hemmern oder anderen Antihypertensiva im ersten Schwangerschaftstrimester (TMS) ist nicht durch die Medikamente, sondern durch die Grundkrankheit Hypertonie verursacht.}

- Das nordkalifornische Kaiser-Permanente-Register enthält alle Daten über Entbindungen, die Diagnosen von Missbildungen, Angaben zur Schwangerschaftshypertonie und die Ausgabe von Medikamenten nach Datum, Dosis und Menge. Die Autoren werteten die Informationen von 465754 Mutter-KindPaaren in den Jahren 1995 bis 2008 aus. Als Vergleich dienten zwei Gruppen, die nie Antihypertensiva erhalten hatten: normotone Kontrollen mit normalem

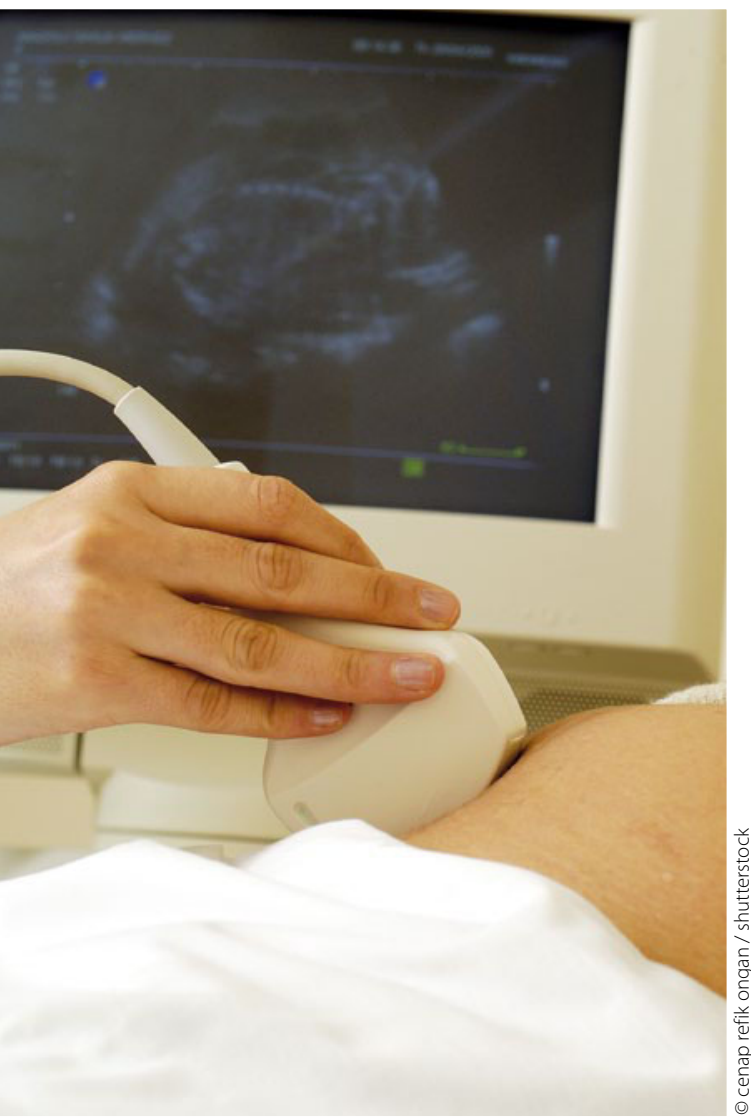

Was ist gefährlicher für den Föten: die Hypertonie oder Antihypertensiva?
Blutdruck vor und während der Schwangerschaft und hypertensive Kontrollen mit erhöhtem Blutdruck, aber ohne antihypertensive Therapie. Für Einflussfaktoren wie Diabetes, Alter, Rasse, Geburtenzahl und Gewicht wurde adjustiert. Ergebnis:

1. Schwangere mit unbehandelter Hypertonie haben gegenüber normotensiven Kontrollen ein um 25\% erhöhtes Missbildungsrisiko (s. Tab. 1).

2. Wenn hypertensive Schwangere im 1. TMS irgendwelche Antihypertensiva oder ACE-Hemmer erhalten, bleibt das
Missbildungsrisiko im Vergleich zu Hypertonikerinnen ohne Behandlung mit 22 bzw. $20 \%$ bzw. 0 bzw. $-3 \%$ unverändert

3. Bei der Analyse für bestimmte Missbildungen. z. B. des Herzens war das Ergebnis identisch, d. h. höheres Risiko im Vergleich zu Normotonikern, aber ähnliches Risiko im Vergleich zu unbehandelten Hypertonikerinnen.

4. Das Missbildungsrisiko bei Gabe von Antihypertensiva und - stärker für ACE-Hemmer - im 2. und 3. TMS ist deutlich erhöht.

\section{Tabelle 1}

Risiko für Geburtsdefekte in Abhängigkeit von Blutdruck und antihypertensiver Therapie (TMS = Trimester)

\begin{tabular}{|l|l|l|}
\hline Normotensive Kontrollen & Referenz 1,00 & - \\
\hline Hypertensive Kontrollen (unbehandelt) & 1,25 & Referenz 1,00 \\
\hline ACE-Hemmer im 1. TMS & 1,20 & 0,97 \\
\hline Andere Antihypertensiva im 1.TMS & 1,22 & 1,00 \\
\hline ACE-Hemmer im 2.+3. TMS & 2,34 & 1,88 \\
\hline Andere Antihypertensiva im 2.+3. TMS & 1,94 & 1,53
\end{tabular}

\section{Kommentar}

Die teratogene Wirkung der ACE-Hemmer im 2. und 3. TMS ist gut belegt, wird von der FDA im genannten Zeitraum als gesichert klassifiziert und wird in dieser Studie bestätigt. Allerdings sind die Daten dieser umfangreichen, methodisch anspruchvollen Studie geeignet, einige andere Glaubenssätze zur antihypertensiven Therapie in der Schwangerschaft zu hinterfragen. Denn das Risiko der anderen, nicht einzeln bewerteten Antihypertensiva ist zwar geringer als das der ACE-Hemmer, aber keineswegs unbedeutend (1,94 vs. 2,34 bzw. 1,53 vs. 1,88).

Praktisch bedeutsam sind zwei Ergebnisse für das 1. TMS. Erstens ist das Risiko für $\mathrm{Ge}$ burtsdefekte unter ACE-Hemmern identisch mit dem durch andere Antihypertensiva. Zweitens liegt das Risiko in beiden Fällen in der gleichen Größenordnung wie bei hypertensiven Schwangeren, die keine Antihyper- tensiva erhalten. Das kann nur bedeuten, dass nicht die Antihypertensiva, sondern die Grundkrankheit Hypertonie das Missbildungsrisiko bestimmt. Man wird abwarten müssen, ob und wie die Fachgesellschaften auf diese neue Botschaft reagieren. Vorerst gibt es eine Beruhigung: Wenn eine Frau unter ACE-Hemmer-Therapie ungeplant schwanger wird oder die Einnahme nicht rechtzeitig beendet, hat man noch drei Monate Zeit bis zum Absetzen. Deshalb scheint auch die Empfehlung überholt, ACE-Hemmer bei Kinderwunsch bzw. im geburtsfähigen Alter strikt zu meiden.

H. HOLZGREVE

\section{- D.-K. Li et al.}

Maternal exposure to angiotensin converting enzyme inhibitors in the first trimester and risk of malformations in offspring: a retrospective cohort study. Brit. Med. J. 2011; 343: d5931 